ANNALES

POLONICI MATHEMATICI

$93.3(2008)$

\title{
Continuous linear functionals on the space of Borel vector measures
}

\author{
by Pola SiwEK (Katowice)
}

\begin{abstract}
We study properties of the space $\mathcal{M}$ of Borel vector measures on a compact metric space $X$, taking values in a Banach space $E$. The space $\mathcal{M}$ is equipped with the Fortet-Mourier norm $\|\cdot\|_{\mathcal{F}}$ and the semivariation norm $\|\cdot\|(X)$. The integral introduced by K. Baron and A. Lasota plays the most important role in the paper. Investigating its properties one can prove that in most cases the space $\left(\mathcal{M},\|\cdot\|_{\mathcal{F}}\right)^{*}$ is contained in but not equal to the space $(\mathcal{M},\|\cdot\|(X))^{*}$. We obtain a representation of the continuous functionals on $\mathcal{M}$ in some particular cases.
\end{abstract}

Introduction. This research was inspired by the paper of K. Baron and A. Lasota [1] in which they studied, among other things, properties of continuous (with respect to the Fortet-Mourier norm) linear functionals on the space $\mathcal{M}$ of vector measures defined on the Borel subsets of a compact metric space $X$ and having values in a real Banach space $E$. They proved that such a functional $\varphi$ is represented by an integral,

$$
\varphi(\mu)=\int_{X} \psi(x, \mu(d x)),
$$

for some function $\psi: X \times E \rightarrow \mathbb{R}$ satisfying certain conditions (see Section 1 for precise definitions), but they did not know whether the converse was true.

We solve this problem under the assumption that $E$ is finite-dimensional. In that case the functional given by $(0.1)$ is continuous with respect to the Fortet-Mourier norm and we obtain another representation of the elements of the space $\left(\mathcal{M},\|\cdot\|_{\mathcal{F}}\right)^{*}$. We also prove that in general every functional given by $(0.1)$ is continuous with respect to the semivariation norm $\|\cdot\|(X)$ and we answer in the negative the natural question whether every functional $\varphi \in(\mathcal{M},\|\cdot\|(X))^{*}$ has the form $(0.1)$.

The paper is organised as follows. In Section 1 we introduce the terminology and preliminary results used throughout the paper. In Section 2 we

2000 Mathematics Subject Classification: Primary 46E27, 28B05; Secondary 46G10. Key words and phrases: Fortet-Mourier norm, vector-valued measures. 
prove the continuity with respect to the semivariation norm of functionals $\varphi$ given by (0.1). The third section contains a proof of the fact that when $X$ is infinite, then there exists a functional $\varphi \in(\mathcal{M},\|\cdot\|(X))^{*}$ which cannot be represented by this formula. The last section solves affirmatively the problem of Baron and Lasota in the case where $E$ is finite-dimensional.

Earlier R. D. Mauldin obtained integral representations for the dual of the space of vector measures taking values in a Banach space with the RadonNikodym property, equipped with the total variation norm. The integral considered by Mauldin is based on Riemann-type sums and the set of partitions is directed by refinement. For more information see [6] and [7].

1. Basic definitions and facts. Throughout this paper $(E,\|\cdot\|)$ will be a nontrivial real Banach space and $(X, \varrho)$ will be a nonempty compact metric space.

Let $\mathcal{B}(X)$ denote the family of Borel subsets of $X$ and let $\mathcal{M}$ denote the linear space of all $\sigma$-additive vector measures $\mu: \mathcal{B}(X) \rightarrow E$. By $C(X)$ we denote the space of all continuous functions $f: X \rightarrow \mathbb{R}$ with the supremum norm $\|f\|_{\infty}$. Define

$$
\operatorname{Lip}_{1}(X)=\{f: X \rightarrow \mathbb{R}|| f(x)-f(z) \mid \leq \varrho(x, z) \text { for all } x, z \in X\} \subseteq C(X) .
$$

In $\mathcal{M}$ we introduce the Fortet-Mourier norm by

$$
\|\mu\|_{\mathcal{F}}=\sup \left\{\left\|\int_{X} f d \mu\right\|: f \in \operatorname{Lip}_{1}(X),\|f\|_{\infty} \leq 1\right\} .
$$

In the case $E=\mathbb{R}$ it follows easily from [4; Proposition 11.3.2] that the above formula defines a norm on $\mathcal{M}$. In the general case the proof can be found in $[1 ;$ p. 219]. The integral appearing here is the Bartle-Dunford-Schwartz integral [3; Definition 1.1.12]; see also [5; IV.10].

The semivariation of $\mu \in \mathcal{M}$ is defined by

$$
\|\mu\|(B)=\sup \left\{|\lambda \mu|(B): \lambda \in E^{*}\right\} \quad \text { for } B \in \mathcal{B}(X),
$$

where $|\lambda \mu|$ is the total variation of the real-valued measure $\lambda \mu$. Note that

$$
\|\mu\|_{\mathcal{F}} \leq\|\mu\|(X) \leq|\mu|(X) \quad \text { for all } \mu \in \mathcal{M},
$$

where $|\mu|$ denotes the variation of $\mu$ (see [3; Definition 1.1.4]). The first inequality follows from the definition of the Bartle-Dunford-Schwartz integral given in [3].

Let us define the notion of integral appearing in (0.1).

Definition 1.1. We say that a function $\psi: X \rightarrow E^{*}$ is integrable with respect to $\mu \in \mathcal{M}$ if there exists a real number $c$ satisfying the following condition $(\mathrm{C})$ : 
(C) For every $\varepsilon>0$ there exists $\delta>0$ such that

$$
\left|\sum_{i=1}^{m} \psi\left(x_{i}\right)\left(\mu\left(B_{i}\right)\right)-c\right|<\varepsilon
$$

for every finite partition $B_{1}, \ldots, B_{m}$ of $X$ into nonempty Borel sets of diameter less than $\delta$ and for all $x_{1} \in B_{1}, \ldots, x_{m} \in B_{m}, m \in \mathbb{N}$.

If $\psi: X \rightarrow E^{*}$ is integrable with respect to $\mu \in \mathcal{M}$, then the integral

$$
\int_{X} \psi(x, \mu(d x))
$$

is defined as the (only) real number $c$ satisfying $(\mathrm{C})$.

The following conditions $\left(\mathrm{A}^{\prime}\right)$ and $(\mathrm{A})$ guarantee (see Lemma 1.1 below) the integrability of a function $\psi: X \rightarrow E^{*}$ with respect to any $\mu \in \mathcal{M}$.

$\left(\mathrm{A}^{\prime}\right)$ For every $\varepsilon>0$ there exists $\delta>0$ such that for any $m \in \mathbb{N}$ and for all $x_{1}, \ldots, x_{m}, z_{1}, \ldots, z_{m} \in X, a_{1}, \ldots, a_{m} \in E$, if

$$
\varrho\left(x_{i}, z_{i}\right) \leq \delta \quad \text { for every } i \in\{1, \ldots, m\},
$$

then

$$
\left|\sum_{i=1}^{m}\left(\psi\left(x_{i}\right) a_{i}-\psi\left(z_{i}\right) a_{i}\right)\right| \leq \varepsilon \sup \left\{\left\|\sum_{i=1}^{m} \varepsilon_{i} a_{i}\right\|:\left|\varepsilon_{1}\right| \leq 1, \ldots,\left|\varepsilon_{m}\right| \leq 1\right\} .
$$

(A) There exists a real constant $L \geq 0$ such that

$$
\left|\sum_{i=1}^{m}\left(\psi\left(x_{i}\right) a_{i}-\psi\left(z_{i}\right) a_{i}\right)\right| \leq L \varrho(x, z)\|a\|
$$

for all $x_{1}, \ldots, x_{m}, z_{1}, \ldots, z_{m} \in X, a_{1}, \ldots, a_{m} \in E$ and $m \in \mathbb{N}$, where

$$
\begin{gathered}
\|a\|:=\sup \left\{\left\|\sum_{i=1}^{m} \varepsilon_{i} a_{i}\right\|:\left|\varepsilon_{1}\right| \leq 1, \ldots,\left|\varepsilon_{m}\right| \leq 1\right\}, \\
\varrho(x, z):=\max \left\{\varrho\left(x_{i}, z_{i}\right): i \in\{1, \ldots, m\}\right\} .
\end{gathered}
$$

Obviously, condition $(\mathrm{A})$ implies $\left(\mathrm{A}^{\prime}\right)$. Moreover, every $\psi: X \rightarrow E^{*}$ satisfying $\left(\mathrm{A}^{\prime}\right)$ is uniformly continuous, hence bounded.

Let $K>0$. We will consider the set

$$
\mathcal{M}_{K}:=\{\mu \in \mathcal{M}:\|\mu\|(X) \leq K\} .
$$

Lemma 1.1. Let $\psi: X \rightarrow E^{*}$ be a function satisfying $\left(\mathrm{A}^{\prime}\right)$. Then $\psi$ is integrable with respect to every $\mu \in \mathcal{M}$. Moreover, given $K>0$ and $\varepsilon>0$, there is $\delta>0$ such that for each partition $B_{1}, \ldots, B_{m}$ of $X$ into nonempty Borel sets of diameter less than $\delta$ and for each choice of points 
$x_{1} \in B_{1}, \ldots, x_{m} \in B_{m}$ we have

$$
\left|\sum_{i=1}^{m} \psi\left(x_{i}\right) \mu\left(B_{i}\right)-\int_{X} \psi(x, \mu(d x))\right| \leq \varepsilon
$$

for all $\mu \in \mathcal{M}_{K}$.

Proof. For any $n \in \mathbb{N}$ let $B_{1}^{n}, \ldots, B_{m_{n}}^{n} \in \mathcal{B}(X)$ be a finite partition of $X$ into nonempty sets of diameter less than $1 / n$ and let $x_{1}^{n} \in B_{1}^{n}, \ldots, x_{m_{n}}^{n}$ $\in B_{m_{n}}^{n}$. We will show that the sequence of real-valued functions

$$
\mu \mapsto \sum_{i=1}^{m_{n}} \psi\left(x_{i}^{n}\right) \mu\left(B_{i}^{n}\right)
$$

defined on $\mathcal{M}_{K}$ satisfies the uniform Cauchy condition and converges uniformly to the function

$$
\mu \mapsto \int_{X} \psi(x, \mu(d x)) .
$$

Fix $\varepsilon>0$ and using $\left(\mathrm{A}^{\prime}\right)$ choose $\delta>0$ suitable for $\varepsilon / 2 K$. For fixed natural numbers $p, q \geq 1 / \delta$ put

$$
\begin{aligned}
B_{i, j} & =B_{i}^{p} \cap B_{j}^{q} \quad \text { for } i \in\left\{1, \ldots, m_{p}\right\}, j \in\left\{1, \ldots, m_{q}\right\}, \\
\Sigma & =\left\{(i, j) \in\left\{1, \ldots, m_{p}\right\} \times\left\{1, \ldots, m_{q}\right\}: B_{i, j} \neq \emptyset\right\}
\end{aligned}
$$

and pick $x_{i, j} \in B_{i, j}$ for any $(i, j) \in \Sigma$. Let $\mu \in \mathcal{M}_{K}$. Then, applying the fact that (see [3; Proposition 1.1.11(a)])

$$
\sup \left\{\left\|\sum_{i=1}^{m_{p}} \sum_{j=1}^{m_{q}} \varepsilon_{i, j} \mu\left(B_{i, j}\right)\right\|:\left|\varepsilon_{1,1}\right| \leq 1, \ldots,\left|\varepsilon_{m_{p}, m_{q}}\right| \leq 1\right\} \leq\|\mu\|(X),
$$

the additivity of $\psi(x)$ for all $x \in X$ and the choice of $\delta$, we obtain

$$
\begin{aligned}
\mid \sum_{i=1}^{m_{p}} \psi\left(x_{i}^{p}\right) \mu\left(B_{i}^{p}\right)- & \sum_{j=1}^{m_{q}} \psi\left(x_{j}^{q}\right) \mu\left(B_{j}^{q}\right) \mid \\
\leq & \left|\sum_{(i, j) \in \Sigma} \psi\left(x_{i}^{p}\right) \mu\left(B_{i, j}\right)-\sum_{(i, j) \in \Sigma} \psi\left(x_{i, j}\right) \mu\left(B_{i, j}\right)\right| \\
& +\left|\sum_{(i, j) \in \Sigma} \psi\left(x_{j}^{q}\right) \mu\left(B_{i, j}\right)-\sum_{(i, j) \in \Sigma} \psi\left(x_{i, j}\right) \mu\left(B_{i, j}\right)\right| \\
\leq & 2 \cdot \frac{\varepsilon}{2 K}\|\mu\|(X) \leq \varepsilon .
\end{aligned}
$$

Now, let $I: \mathcal{M}_{K} \rightarrow \mathbb{R}$ denote the uniform limit of the sequence (1.2). Fix $\varepsilon>0$. Apply $\left(\mathrm{A}^{\prime}\right)$ to choose $\delta>0$ suitable for $\varepsilon / 4 K$ and take a natural 
number $n \geq 1 / \delta$ such that

$$
\left|\sum_{j=1}^{m_{n}} \psi\left(x_{j}^{n}\right) \mu\left(B_{j}^{n}\right)-I(\mu)\right| \leq \varepsilon / 2 \quad \text { for all } \mu \in \mathcal{M}_{K} .
$$

Let $\mu \in \mathcal{M}_{K}$. Fix a partition $B_{1}, \ldots, B_{m}$ of $X$ into nonempty Borel sets of diameter less than $\delta$ and pick $x_{1} \in B_{1}, \ldots, x_{m} \in B_{m}$. Setting

$$
\begin{aligned}
B_{i, j} & =B_{i} \cap B_{j}^{n} \quad \text { for } i \in\{1, \ldots, m\}, j \in\left\{1, \ldots, m_{n}\right\}, \\
\Sigma & =\left\{(i, j) \in\{1, \ldots, m\} \times\left\{1, \ldots, m_{n}\right\}: B_{i, j} \neq \emptyset\right\},
\end{aligned}
$$

choosing any $x_{i, j} \in B_{i, j}$ for every $(i, j) \in \Sigma$ and arguing as above we obtain

$$
\begin{aligned}
\mid \sum_{i=1}^{m} \psi\left(x_{i}\right) \mu\left(B_{i}\right) & -I(\mu) \mid \\
\leq & \left|\sum_{i=1}^{m} \psi\left(x_{i}\right) \mu\left(B_{i}\right)-\sum_{j=1}^{m_{n}} \psi\left(x_{j}^{n}\right) \mu\left(B_{j}^{n}\right)\right|+\varepsilon / 2 \\
\leq & \left|\sum_{(i, j) \in \Sigma} \psi\left(x_{i}\right) \mu\left(B_{i, j}\right)-\sum_{(i, j) \in \Sigma} \psi\left(x_{i, j}\right) \mu\left(B_{i, j}\right)\right| \\
& +\left|\sum_{(i, j) \in \Sigma} \psi\left(x_{j}^{n}\right) \mu\left(B_{i, j}\right)-\sum_{(i, j) \in \Sigma} \psi\left(x_{i, j}\right) \mu\left(B_{i, j}\right)\right|+\frac{\varepsilon}{2} \\
\leq & 2 \cdot \frac{\varepsilon}{4 K}\|\mu\|(X)+\frac{\varepsilon}{2} \leq \varepsilon .
\end{aligned}
$$

We have shown that for all $\mu \in \mathcal{M}_{K}$ the real number $I(\mu)$ satisfies $(\mathrm{C})$ and so $\psi$ is integrable with respect to $\mu$, with integral $I(\mu)$. Since

$$
\mathcal{M}=\bigcup_{K>0} \mathcal{M}_{K}
$$

this completes the proof of the lemma.

Comments. As observed by the referee, in the case of a countably additive vector measure having finite variation one can compare the integral (1.1) with the Bartle bilinear *-integral [2] considered with respect to the natural bilinear mapping

$$
(\lambda, a) \mapsto \lambda a, \quad(\lambda, a) \in E^{*} \times E .
$$

Fix $\mu \in \mathcal{M}$ with $|\mu|(X)<\infty$. Clearly, the semivariation of $\mu$ in the sense of [2] with respect to the mapping (1.3) is finite (see [2; pp. 338-339]). We will show that if a function $\psi: X \rightarrow E^{*}$ satisfies $\left(\mathrm{A}^{\prime}\right)$, then it is $\mu$-integrable in the sense of Bartle [2; Definition 1] and the Bartle $*$-integral of $\psi$ with respect to $\mu$ is just the integral (1.1) defined in Definition 1.1.

Indeed, for every $n \in \mathbb{N}$ let $B_{1}^{n}, \ldots, B_{m_{n}}^{n} \in \mathcal{B}(X)$ be a finite partition of $X$ into nonempty sets of diameter less than $1 / n$ and pick $x_{1}^{n} \in B_{1}^{n}, \ldots$, 
$x_{m_{n}}^{n} \in B_{m_{n}}^{n}$. Then, by the uniform continuity of $\psi$ and the finiteness of the variation of $\mu$, the sequence

$$
\left(\sum_{i=1}^{m_{n}} \psi\left(x_{i}^{n}\right) \cdot \mathbb{1}_{B_{i}^{n}}\right)_{n \in \mathbb{N}}
$$

of $\mu$-simple functions satisfies all the requirements of [2; Definition 1] and by [2; Theorem 1] the function $\psi$ is $\mu$-integrable in the sense of Bartle and its Bartle *-integral is exactly the limit

$$
\lim _{n \rightarrow \infty} \sum_{i=1}^{m_{n}} \psi\left(x_{i}^{n}\right) \mu\left(B_{i}^{n}\right) .
$$

But in the proof of Lemma 1.1 we showed that the above limit equals the integral (1.1).

We will now state the aforementioned result of Baron and Lasota, viz. [1; Theorem 2.1] and [1; Corollary 2.1]. Define

$$
\delta_{x}(B)=\mathbb{1}_{B}(x) \quad \text { for } x \in X \text { and } B \in \mathcal{B}(X) .
$$

Clearly $a \delta_{x} \in \mathcal{M}$ for any $a \in E$.

THEOREM 1.1 (Baron-Lasota). If $\varphi$ is a continuous linear functional on $\left(\mathcal{M},\|\cdot\|_{\mathcal{F}}\right)$, then the function $\psi: X \rightarrow E^{*}$ defined by

$$
\psi(x) a=\varphi\left(a \delta_{x}\right)
$$

satisfies (A); moreover,

$$
\varphi(\mu)=\int_{X} \psi(x, \mu(d x)) \quad \text { for all } \mu \in \mathcal{M} .
$$

The set

$$
\mathcal{M}_{\text {fin }}:=\{\mu \in \mathcal{M}:|\mu|(X)<\infty\}
$$

is a linear subspace of $\mathcal{M}$.

COROLlary 1.1 (Baron-Lasota). If $\varphi$ is a continuous linear functional on $\left(\mathcal{M}_{\text {fin }},\|\cdot\|_{\mathcal{F}}\right)$, then the function $\psi: X \rightarrow E^{*}$ defined by (1.4) satisfies (A); moreover,

$$
\varphi(\mu)=\int_{X} \psi(x, \mu(d x)) \quad \text { for all } \mu \in \mathcal{M}_{\text {fin }}
$$

2. Continuity with respect to the semivariation norm. Our main result reads as follows.

Theorem 2.1. Let $\psi: X \rightarrow E^{*}$ satisfy $\left(\mathrm{A}^{\prime}\right)$. Then the functional $\varphi$ : $\mathcal{M} \rightarrow \mathbb{R}$ defined by

$$
\varphi(\mu)=\int_{X} \psi(x, \mu(d x))
$$

is linear and continuous with respect to the semivariation norm $\|\cdot\|(X)$. 
Proof. The linearity of $\varphi$ is straightforward. It remains to verify the continuity of $\varphi$ at zero.

Let $\left(\mu_{n}\right)_{n \in \mathbb{N}}$ be a sequence in $\mathcal{M}$ which converges to zero in the semivariation norm. Evidently, there exists a positive number $K$ such that

$$
\left\{\mu_{n}: n \in \mathbb{N}\right\} \subseteq \mathcal{M}_{K} .
$$

Fix $\varepsilon>0$, choose $\delta>0$ as in Lemma 1.1, fix a partition $B_{1}, \ldots, B_{m}$ of $X$ into nonempty Borel sets of diameter less than $\delta$ and select $x_{1} \in B_{1}, \ldots$, $x_{m} \in B_{m}$. Then, by the boundedness of $\psi$ (see remarks after Definition 1.2) and [2; Proposition 1.1.11(b)], for any $n \in \mathbb{N}$ we have

$$
\begin{aligned}
\left|\int_{X} \psi\left(x, \mu_{n}(d x)\right)\right| & \leq \varepsilon+\sum_{i=1}^{m}\left|\psi\left(x_{i}\right) \mu_{n}\left(B_{i}\right)\right| \\
& \leq \varepsilon+\sup _{x \in X}\|\psi(x)\|_{E^{*}} \sum_{i=1}^{m}\left\|\mu_{n}\left(B_{i}\right)\right\| \\
& \leq \varepsilon+m \sup _{x \in X}\|\psi(x)\|_{E^{*}}\left\|\mu_{n}\right\|(X),
\end{aligned}
$$

and consequently

$$
\limsup _{n \rightarrow \infty}\left|\int_{X} \psi\left(x, \mu_{n}(d x)\right)\right| \leq \varepsilon
$$

As immediate consequences of Theorems 1.1 and 2.1 we have the following corollaries.

Corollary 2.1. If $\psi: X \rightarrow E^{*}$ satisfies $\left(\mathrm{A}^{\prime}\right)$, then the linear functional $\varphi: \mathcal{M}_{\text {fin }} \rightarrow \mathbb{R}$ defined by (2.1) is continuous with respect to the variation norm $|\cdot|(X)$.

COROllary 2.2. The following inclusions hold:

$$
\begin{aligned}
\left(\mathcal{M},\|\cdot\|_{\mathcal{F}}\right)^{*} & \subseteq\{\varphi: \mathcal{M} \rightarrow \mathbb{R}: \varphi \text { is defined by }(2.1) \text { with } \\
& \text { a } \left.\psi: X \rightarrow E^{*} \text { satisfying }\left(\mathrm{A}^{\prime}\right)\right\} \\
& \subseteq(\mathcal{M},\|\cdot\|(X))^{*}
\end{aligned}
$$

and

$$
\begin{aligned}
&\left(\mathcal{M}_{\text {fin }},\|\cdot\|_{\mathcal{F}}\right)^{*} \subseteq\left\{\varphi: \mathcal{M}_{\text {fin }} \rightarrow \mathbb{R}: \varphi \text { is defined by }(2.1)\right. \text { with } \\
&\left.\qquad a \psi: X \rightarrow E^{*} \text { satisfying }\left(\mathrm{A}^{\prime}\right)\right\} \\
& \subseteq\left(\mathcal{M}_{\mathrm{fin}},\|\cdot\|(X)\right)^{*} .
\end{aligned}
$$

The question arises whether, in the above chains of inclusions, the second inclusion can be replaced by an equality. In the next section we show that the answer is negative in general. 
3. More about the structure of $(\mathcal{M},\|\cdot\|(X))^{*}$. The purpose of this section is to prove the following theorem.

Theorem 3.1. If $X$ is infinite, then there exists a $\varphi \in(\mathcal{M},\|\cdot\|(X))^{*}$ which does not admit a representation of the form (1.5) for any function $\psi: X \rightarrow E^{*}$ satisfying condition $\left(\mathrm{A}^{\prime}\right)$.

Actually, one can obtain a stronger statement. For this we formally extend Definition 1.1 to cover functions $\psi: X \rightarrow \mathbb{R}^{E}$.

Definition 3.1. We say that a function $\psi: X \rightarrow \mathbb{R}^{E}$ is integrable with respect to $\mu \in \mathcal{M}$ if there exists a real number $c$ satisfying $(\mathrm{C})$.

If $\psi: X \rightarrow \mathbb{R}^{E}$ is integrable with respect to $\mu \in \mathcal{M}$, then the integral (1.1) is defined as the (only) real number $c$ satisfying (C).

We start with the following lemma.

Lemma 3.1. If $\psi: X \rightarrow \mathbb{R}^{E}$ is integrable with respect to every $\mu \in \mathcal{M}_{\text {fin }}$ in the sense of Definition 3.1 and

$$
\psi(x)(0)=0 \quad \text { for all } x \in X,
$$

then for every $a \in E$ the function $\psi(\cdot)(a)$ is continuous and

$$
\int_{X} \psi\left(x, a \delta_{z}(d x)\right)=\psi(z)(a) \quad \text { for all } z \in X \text { and } a \in E .
$$

Proof. To prove the first statement fix $a \in E, x_{0} \in X, \varepsilon>0$ and, applying condition (C) to the measure $a \delta_{x_{0}}$, choose a $\delta>0$ suitable for $\varepsilon / 2$.

Let $z \in X$ be such that $\varrho\left(z, x_{0}\right)<\delta / 2$. Choosing a finite partition $B_{1}, \ldots, B_{m} \in \mathcal{B}(X)$ of $X$ into nonempty sets of diameter less than $\delta$ such that $\left\{x_{0}, z\right\} \subseteq B_{1}$ and picking $x_{2} \in B_{2}, \ldots, x_{m} \in B_{m}$, we have

$$
\begin{aligned}
& \left|\psi\left(x_{0}\right)(a)-\psi(z)(a)\right| \\
& \leq\left|\psi\left(x_{0}\right)\left(a \delta_{x_{0}}\left(B_{1}\right)\right)+\sum_{i=2}^{m} \psi\left(x_{i}\right)\left(a \delta_{x_{0}}\left(B_{i}\right)\right)-\int_{X} \psi\left(x, a \delta_{x_{0}}(d x)\right)\right| \\
& \quad+\left|\psi(z)\left(a \delta_{x_{0}}\left(B_{1}\right)\right)+\sum_{i=2}^{m} \psi\left(x_{i}\right)\left(a \delta_{x_{0}}\left(B_{i}\right)\right)-\int_{X} \psi\left(x, a \delta_{x_{0}}(d x)\right)\right|<\varepsilon .
\end{aligned}
$$

The second statement follows easily from the continuity of $\psi(\cdot)(a)$ at $z$.

The stronger statement mentioned after Theorem 3.1 reads as follows.

THEOREM 3.2. If $X$ is infinite, then there exists a $\varphi \in\left(\mathcal{M}_{\mathrm{fin}},\|\cdot\|(X)\right)^{*}$ which does not admit a representation of the form (1.6) for any function $\psi: X \rightarrow \mathbb{R}^{E}$ satisfying (3.1) and integrable with respect to every $\mu \in \mathcal{M}_{\text {fin }}$ in the sense of Definition 3.1. 
Proof. For any $a \in E \backslash\{0\}$ we will find a $\varphi \in\left(\mathcal{M}_{\text {fin }},\|\cdot\|(X)\right)^{*}$ such that the function

$$
x \mapsto \varphi\left(a \delta_{x}\right), \quad x \in X,
$$

is discontinuous. This jointly with Lemma 3.1 will prove the theorem.

Let $z \in X$ be an accumulation point of $X$ and fix any $a \in E \backslash\{0\}$. Obviously, the set

$$
M=\left\{\sum_{i=1}^{m} a_{i} \delta_{x_{i}}: a_{1}, \ldots, a_{m} \in E ; x_{1}, \ldots, x_{m} \in X \backslash\{z\} ; m \in \mathbb{N}\right\}
$$

is a linear subspace of $\mathcal{M}_{\text {fin }}$ and for any $a_{1}, \ldots, a_{m} \in E, x_{1}, \ldots, x_{m} \in X \backslash\{z\}$ we have (according to [2; Proposition 1.1.11(b)])

$$
\left\|a \delta_{z}-\sum_{i=1}^{m} a_{i} \delta_{x_{i}}\right\|(X) \geq\left\|\left(a \delta_{z}-\sum_{i=1}^{m} a_{i} \delta_{x_{i}}\right)(\{z\})\right\|=\|a\|>0,
$$

which shows that

$$
\inf \left\{\left\|\mu-a \delta_{z}\right\|(X): \mu \in M\right\}>0 .
$$

Consequently (see [5; Lemma II.3.12]), there exists a $\varphi \in\left(\mathcal{M}_{\text {fin }},\|\cdot\|(X)\right)^{*}$ such that

$$
\varphi_{\mid M}=0 \quad \text { and } \quad \varphi\left(a \delta_{z}\right)=1 .
$$

Clearly the function (3.2) is discontinuous at $z$.

Corollary 3.1. If $X$ is infinite, then

$$
\begin{gathered}
\left(\mathcal{M},\|\cdot\|_{\mathcal{F}}\right)^{*} \subsetneq(\mathcal{M},\|\cdot\|(X))^{*}, \\
\left(\mathcal{M}_{\text {fin }},\|\cdot\|_{\mathcal{F}}\right)^{*} \subsetneq\left(\mathcal{M}_{\text {fin }},\|\cdot\|(X)\right)^{*} .
\end{gathered}
$$

We finish this section with the following remark showing that in the statements of Theorems 3.1 and 3.2 the assumption on $X$ cannot be dropped:

REMARK 3.1. If a compact space $X$ is made up of isolated points, then it is finite:

$$
X=\left\{x_{1}, \ldots, x_{m}\right\} .
$$

Furthermore, if $E$ is finite-dimensional and the vectors $e_{1}, \ldots, e_{n}$ form a basis of $E$, then the vectors $e_{k} \delta_{x_{l}}$ for $k \in\{1, \ldots, n\}, l \in\{1, \ldots, m\}$ form a basis of $\mathcal{M}$. Hence all norms on $\mathcal{M}$ are equivalent.

4. The case of finite dimension. Now we present a solution of the main problem under the assumption that $E$ is finite-dimensional.

Theorem 4.1. Suppose $E$ is finite-dimensional. If $\psi: X \rightarrow E^{*}$ satisfies (A), then the functional $\varphi: \mathcal{M} \rightarrow \mathbb{R}$ defined by (2.1) is continuous with respect to the Fortet-Mourier norm. 
Proof. Let $N:=\operatorname{dim} E$. Find unit vectors $e_{1}, \ldots, e_{N}$ which form a basis of $E$. Let $\pi_{1}, \ldots, \pi_{N}: E \rightarrow \mathbb{R}$ be the coordinate functionals, i.e.,

$$
a=\sum_{k=1}^{N} \pi_{k}(a) e_{k} \quad \text { for all } a \in E .
$$

Let $L$ be the constant given in condition (A). Setting

$$
f_{k}=\psi(\cdot)\left(e_{k}\right) \quad \text { for } k \in\{1, \ldots, N\},
$$

we have

$$
\psi(x)(a)=\sum_{k=1}^{N} \pi_{k}(a) f_{k}(x) \quad \text { for all } x \in X \text { and } a \in E .
$$

Fix $k \in\{1, \ldots, N\}$. Clearly, $f_{k}$ is Lipschitz with constant $L$. A simple computation now shows that the $E^{*}$-valued mapping

$$
x \mapsto f_{k}(x) \pi_{k}, \quad x \in X,
$$

satisfies condition (A), and hence is integrable with respect to any $\mu \in \mathcal{M}$. We will prove that the integral is $\pi_{k}\left(\int_{X} f_{k} d \mu\right)$. This jointly with (4.1) will show that

$$
\int_{X} \psi(x, \mu(d x))=\sum_{k=1}^{N} \pi_{k}\left(\int_{X} f_{k} d \mu\right) .
$$

Let $\mu \in \mathcal{M}$. Fix $\varepsilon>0$ and choose a $\delta>0$ such that $\delta L\left\|\pi_{k}\right\|\|\mu\|(X)<\varepsilon$. Let $B_{1}, \ldots, B_{m} \in \mathcal{B}(X)$ be a partition of $X$ into nonempty sets of diameter less than $\delta$ and fix $x_{1} \in B_{1}, \ldots, x_{m} \in B_{m}$.

If $z \in X=\bigcup_{i=1}^{m} B_{i}$, then $z \in B_{i}$ for exactly one $i \in\{1, \ldots, m\}$, whence

$$
\left|\sum_{i=1}^{m} f_{k}\left(x_{i}\right) \mathbb{1}_{B_{i}}(z)-f_{k}(z)\right| \leq L \varrho\left(x_{i}, z\right)<L \delta .
$$

Consequently,

$$
\begin{aligned}
& \left|\sum_{i=1}^{m} f_{k}\left(x_{i}\right) \pi_{k}\left(\mu\left(B_{i}\right)\right)-\pi_{k}\left(\int_{X} f_{k} d \mu\right)\right| \\
& \quad=\left|\pi_{k}\left(\int_{X}\left(\sum_{i=1}^{m} f_{k}\left(x_{i}\right) \mathbb{1}_{B_{i}}\right) d \mu\right)-\pi_{k}\left(\int_{X} f_{k} d \mu\right)\right| \\
& \leq\left\|\pi_{k}\right\| \sup \left\{\left|\sum_{i=1}^{m} f_{k}\left(x_{i}\right) \mathbb{1}_{B_{i}}(z)-f_{k}(z)\right|: z \in X\right\}\|\mu\|(X) \\
& \leq\left\|\pi_{k}\right\| L \delta\|\mu\|(X)<\varepsilon .
\end{aligned}
$$

By (4.2) we can rewrite (2.1) as

$$
\varphi(\mu)=\sum_{k=1}^{N} \pi_{k}\left(\int_{X} f_{k} d \mu\right)
$$


for all $\mu \in \mathcal{M}$. Hence with

$$
M:=\max \left\{L,\left\|f_{1}\right\|_{\infty}, \ldots,\left\|f_{N}\right\|_{\infty}\right\}
$$

we obtain

$$
|\varphi(\mu)| \leq \sum_{k=1}^{N}\left\|\pi_{k}\right\| M\|\mu\|_{\mathcal{F}} \quad \text { for all } \mu \in \mathcal{M},
$$

which shows that $\varphi$ is continuous.

We finish with the following representation theorem, which is a consequence of Theorem 1.1 and the proof of Theorem 4.1.

ThEOREM 4.2. If $E$ is finite-dimensional with $\operatorname{dim} E=N$, then for every $\varphi \in\left(\mathcal{M},\|\cdot\|_{\mathcal{F}}\right)^{*}$ there exist functionals $\lambda_{1}, \ldots, \lambda_{N} \in E^{*}$ and Lipschitzian functions $f_{1}, \ldots, f_{N}: X \rightarrow \mathbb{R}$ such that

$$
\varphi(\mu)=\sum_{k=1}^{N} \lambda_{k}\left(\int_{X} f_{k} d \mu\right) \quad \text { for } \mu \in \mathcal{M} .
$$

Conversely, every functional $\varphi$ defined as above is continuous with respect to the Fortet-Mourier norm.

Acknowledgments. The author is grateful to Professor Karol Baron for his valuable advice and essential help.

\section{References}

[1] K. Baron and A. Lasota, Markov operators on the space of vector measures; coloured fractals, Ann. Polon. Math. 69 (1998), 217-234.

[2] R. G. Bartle, A general bilinear vector integral, Studia Math. 15 (1956), 337-352.

[3] J. Diestel and J. J. Uhl, Jr., Vector Measures, Math. Surveys 15, Amer. Math. Soc. 1977.

[4] R. M. Dudley, Real Analysis and Probability, Wadsworth \& Brooks/Cole, 1989.

[5] N. Dunford and J. T. Schwartz, Linear Operators. Part I: General Theory, Interscience, 1958.

[6] R. D. Mauldin, The continuum hypothesis, integration and duals of measure spaces, Illinois J. Math. 19 (1975), 33-44.

[7] —, Some effects of set-theoretical assumptions in measure theory, Adv. Math. 27 (1978), 45-62.

Institute of Mathematics

University of Silesia

Bankowa 14

40-007 Katowice, Poland

E-mail: p_siwek@interia.eu 